\title{
Intercoat Adhesion Failure in a Multilayer Organic Coating System; An X-ray Photoelectron Spectroscopy Study.
}

Steven J. Hinder ${ }^{1 *}$, Chris Lowe ${ }^{2}$, James T. Maxted ${ }^{2}$, Christian Perruchot $^{1 \#}$, John F. Watts ${ }^{1}$.

${ }^{1}$ The Surface Analysis Laboratory, School of Engineering, University of Surrey, Guildford, Surrey GU2 7XH, UK.

${ }^{2}$ Becker Industrial Coatings Ltd, Goodlass Road, Speke, Liverpool L24 9HJ, UK.

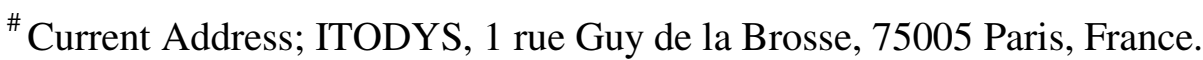

* Correspondence to: Steven J. Hinder, The Surface Analysis Laboratory, School of Engineering, Mail Stop H6, University of Surrey, Guildford, Surrey GU2 7XH, UK.

Email: s.hinder@surrey.ac.uk Fax: +44 (0) 1483686291.

Contract/grant sponsor: EPSRC; Contract/grant number: GR/N65745. 


\section{Abstract :}

The strength of intercoat adhesion exhibited between a series of polyester/polyurethane (PU) based primer formulations and a standard poly(vinylidene difluoride) (PVdF) based topcoat formulation has been investigated by X-ray photoelectron spectroscopy (XPS). An initial XPS study of changes in surface elemental composition (induced by variation of the peak metal temperature (PMT) achieved during thermal curing), on a subset of the PU primers employed, indicates that beyond a PMT of $232^{\circ} \mathrm{C}$ changes in PU primer surface composition are negligible. A reference PU primer coating formulation and four variations of this formulation, produced by including, excluding or substituting components/additives in the reference formulation, are characterised by XPS. The PU primer formulation in which a flow agent additive is included exhibits segregation of the flow agent to the primer surface. The PU primer and PVdF topcoat intercoat adhesion failure surfaces resulting from failure at or near the $\mathrm{PVdF} / \mathrm{PU}$ interface as a result of a peel test are also characterised by XPS. Additionally the PVdF topcoat air-coating surface is characterised by XPS. The interface analyses for the flow agent containing PU primer formulation indicates stripping of the flow agent layer from the PU primer and transfer of the flow agent to the PVdF topcoat interfacial failure surface. Similarly, PU primer formulations in which the concentrations of a crosslinking resin are changed demonstrate that the transfer of carbon and oxygen containing materials from the PU primer to the PVdF topcoat occurs, due to insufficient crosslinking of the polyester component of the PU primer formulation. These results suggest a correlation between the nitrogen concentration at the PU primer surface and the 
strength of the intercoat adhesion exhibited by the PU primer towards the PVdF topcoat.

\section{Keywords :}

Intercoat adhesion failure; Polymeric coatings; Multilayer Organic coatings; X-ray photoelectron spectroscopy.

\section{Running Headline :}

Intercoat Adhesion Failure in a Multilayer Coating; An XPS Study 


\section{Introduction.}

The adhesion of polymers and polymeric based coatings and paints to a variety of substrates is of worldwide industrial and commercial importance. The use of polymer based coatings on substrates as diverse as wood [1], concrete [2,3], glass [4], oxides [5] and metals [6] has been the centre of much research interest. In particular, polymeric coatings on metal substrates have wide ranging applications including architectural (coil coating) [7], aerospace [8], automotive [9], marine [10,11], medical implant [12] and microelectronics [13] industries.

The use of some organic coating systems when applied to metal substrates such as steel and aluminium, only require the application of a single coat; however, many commercial coating systems require a two-step application procedure. Typically a primer is applied directly to the metallic substrate to provide protection against corrosion but also to provide a sound base with good adhesion for the application of a topcoat. The topcoat then provides the properties desirable in a coating that interacts with the environment. These properties include resistance to weathering and water penetration. Although many of the processes associated with the application and curing of polymeric coatings such as component/additive segregation to the coating surface [14-16], characterisation of curing temperature effects [17] and the evaluation of flow agent additive layer thickness [18] have been investigated, the phenomena of intercoat adhesion (which is critically important in the long term effectiveness of multilayer coating procedures) has received limited attention. 
The majority of studies reported in the literature regarding the investigation of polymer-polymer interfaces have concentrated on the structure and chemistry of interfaces formed between polymer blends, be they miscible or immiscible. [19] There is however some literature concerning intercoat adhesion between multilayer polymeric coating/paint systems. Lowe et al. evaluated three complementary methods used to quantify the adhesion of polymeric paints on metals used in the coil coating industry [20]. Lauke et al. have employed a novel curved interface tensile test instrument to examine the adhesion strength exhibited by two polymers towards each other. [21] However, much of the coating/coating adhesion research interest is stimulated by the desire of the automotive manufacturing industry to improve the finish available on motor vehicles [9,22] and motor vehicle components. [23]

In the work described here changes in the strength of intercoat adhesion exhibited by five variants of a PU based primer coating towards a standard PVdF based topcoat formulation have been investigated. XPS has been employed to determine the surface elemental composition of the five PU primer variants employed. The technique has also been used to examine the PU primer and PVdF topcoat adhesion failure surfaces resulting from intercoat adhesion failure at the PVdF/PU coatings interface. The failure occurred when a peel test was applied to initiate disbonding and delamination of the PVdF topcoat from the underlying PU primer. 


\section{Experimental.}

\subsection{Sample Preparation.}

Due to reasons of commercial confidentiality only generic terms will be employed to describe the components and additives included in the PU primer and PVdF topcoat formulations.

The reference model PU primer and its variants and the model PVdF topcoat samples applied to a hot-dipped galvanised steel (HDGS) substrate, employed here as a model, multilayer coating system, were prepared at Becker Industrial Coatings Ltd. The major components of the PU primer reference formulation are hydroxy functional phthalate based polyester resins and both caprolactam and methylethylketoxime blocked isocyanates. Additional components of the PU formulation include pigments giving opacity and corrosion resistance (strontium chromate) and a filler. Minor additives of the PU primer reference formulation include an organotin catalyst. The variations employed in the formulation of the PU primers analysed are described in Table 1. All primer formulations were applied with a bar coater at a $24 \mu \mathrm{m}$ wet film thickness $(5 \mu \mathrm{m}$ dry film) and cured with an oven dwell time of $45 \mathrm{~s}$. All primer only coatings were double baked and quenched immediately in demineralised water.

A single, standard PVdF topcoat formulation was employed. The PVdF topcoat formulation major components are a vinylidene difluoride resin, methyl methacrylate based acrylic co-resins and blue and white pigments. Minor additives to the PVdF 
topcoat formulation include an acrylic flow agent and a matting agent. The PVdF topcoat was applied to provide a $20-22 \mu \mathrm{m}$ dry film thickness and cured at a PMT of $249^{\circ} \mathrm{C}$ with an oven dwell time of $55 \mathrm{~s}$.

For the XPS surface characterisation of the PU primer surfaces, a disc $\sim 1 \mathrm{~cm}$ in diameter was punched from the sample panel and analysed immediately. For the PU primer-PVdF topcoat intercoat adhesion failure surfaces a disc $\sim 1 \mathrm{~cm}$ in diameter was punched from the sample panel. A double cross-hatch pattern (pitch $\sim 2 \mathrm{~mm}$ ) was then scored through the coatings with a scalpel and a peel test performed using adhesive tape. The freshly exposed PU primer adhesion failure surface was analysed immediately taking care to analyse within the cross-hatch scribe lines. Regions of the delaminated PVdF topcoat were removed, where possible, with tweezers and analysed immediately. Where it was not possible to remove the PVdF topcoat with tweezers sections of the PVdF topcoat that remained adhered to the adhesive tape after the peel test were analysed.

\subsection{Adhesive Peel Test.}

To perform a semi-quantitative ECCA/NCCA adhesive test a PVdF topcoat/PU primer on HDGS substrate specimen was scored with $10 \times 10$ cross hatched parallel lines to produce 100 coating squares. Adhesive tape (Scotch 610) was then applied to the cross hatched coating surface and pressure applied to the adhesive tape to ensure a strong adhesive bond existed between the adhesive tape and PVdF topcoat air-coating surface. The adhesive tape was then removed in a single, rapid motion to attempt to 
delaminate the PVdF topcoat from the PU primer. The number of delaminated coating squares was then recorded to determine the \% adhesion of the PVdF coating.

\subsection{X-ray Photoelectron Spectroscopy.}

XPS analyses were performed on a Thermo VG Scientific Sigma Probe spectrometer (Thermo VG Scientific, East Grinstead, UK). The instrument is equipped with both a microfocus monochromated AlK $\alpha$ source and a standard twin anode source $(\mathrm{AlK} \alpha / \mathrm{MgK} \alpha)$. In the current work both the twin anode $\mathrm{AlK} \alpha \mathrm{X}$-ray source $(\mathrm{h} v=$ $1486.6 \mathrm{eV})$ used at $300 \mathrm{~W}(15 \mathrm{kV} \times 20 \mathrm{~mA})$ and the monochromated AlKa X-ray source $(\mathrm{hv}=1486.6 \mathrm{eV})$ used at $140 \mathrm{~W}$ were employed. The area of analysis was approximately $800 \mu \mathrm{m}$ diameter for the twin anode source and $500 \mu \mathrm{m}$ diameter for the monochromated source. The pass energy was set at $20 \mathrm{eV}$ for high resolution, core level spectra of all elements of interest. The coating samples were held in place on the instruments sample stage by a sprung $\mathrm{Cu} / \mathrm{Be}$ clip. Quantitative surface chemical analyses were calculated from the high resolution core level spectra, following the removal of a non-linear background. The manufacturer's Avantage software was used which incorporates the appropriate sensitivity factors and corrects for the electron energy analyser transmission function. 


\section{Results and Discussion.}

In the studies described here the surface elemental composition and adhesion properties exhibited by five variants of a PU primer coating formulation have been investigated. The variations in the PU primer coating formulations employed are described in Table 1. As shown in Table 1 components of the PU primer formulation are changed (E2 \& E3), or components added (E4 \& E5) to induce changes in the properties exhibited by the PU primer surfaces. Table 2 presents the results from an investigation into the strength of adhesion exhibited by the PU primer/PVdF topcoat system, as assessed by the adhesive tape peel test. The PU primers that exhibited the poorest adhesion between the PU primer and PVdF topcoat displayed $0 \%$ adhesion. Typically in primers achieving 0\% adhesion, upon curing of the PVdF topcoat to the primer, the topcoat readily delaminates and can be easily removed in large sections by the use of tweezers prior to application of the adhesive tape test. PU primers achieving $100 \%$ adhesion display an adhesive strength so great that it is not possible to disbond the PVdF topcoat from the PU primer even upon application of the adhesive tape. Although none of the PU primer formulations described in these studies achieved $100 \%$ adhesion, when commercial formulations were tested in a similar manner it proved impossible to disbond and delaminate the commercial PVdF topcoat from the underlying PU primer.

In an initial study to determine a common curing temperature for the primer formulations employed, a subset of the PU primers (E3 \& E4) were cured at four different PMTs to investigate changes in surface elemental composition resulting 
from the use of different curing temperatures. In Figure 1 an XPS survey spectra acquired for the E3 formulation cured at $224^{\circ} \mathrm{C}$ is presented. XPS signals characteristic of $\mathrm{C} 1 \mathrm{~s}(\sim 285 \mathrm{eV}), \mathrm{O} 1 \mathrm{~s}(\sim 533 \mathrm{eV}), \mathrm{N} 1 \mathrm{~s}(\sim 400 \mathrm{eV}), \mathrm{Sn} 3 \mathrm{~d}(\sim 486 \&$ $494 \mathrm{eV})$ and $\mathrm{Sn} 3 \mathrm{p}(\sim 715 \& 757 \mathrm{eV})$ are observed. The XPS survey spectra in Figure 1 is typical of that observed for both primers at all PMTs in this initial study. In Table 3 the elemental surface compositions of PU primer formulations E3 and E4 cured at PMTs of $224^{\circ} \mathrm{C}, 232^{\circ} \mathrm{C}, 242^{\circ} \mathrm{C}$ and $249^{\circ} \mathrm{C}$ are presented.

It is observed in Table 3 that the surface concentration (in atomic \%) of carbon and oxygen are very similar for both primer formulations regardless of the PMT curing temperature employed. Additionally, Table 3 shows that for both of the PU primer formulations investigated, raising the PMT curing temperature leads to an increase in the surface concentration of nitrogen. The concentration of tin remains similar for both primers regardless of the PMT curing temperature employed. The tin species present in the PU primer arise from the addition of an organotin curing catalyst to the formulation.

As already discussed, Table 3 demonstrates that the surface concentration of tin for the E3 and E4 PU primer surfaces are similar. At PMTs above $232^{\circ} \mathrm{C}$ the surface concentrations of tin in the E3 and E4 PU primers are very similar. The similarity in tin concentrations above $232^{\circ} \mathrm{C}$ for the E3 and E4 PU primers indicates that the addition of the matting agent to the PU primer formulations does not affect the surface composition of the primer. Furthermore, the similarity in tin concentrations 
shows that the matting agent, when added to the E3 PU primer formulation to make E4, is not expressed at the PU primer surface.

The principal source of the nitrogen species in both PU primer formulations is the crosslinking agent employed. The increase in nitrogen concentration at the surface of the PU primers in Table 3 (associated with increasing PMT curing temperature), suggests the crosslinking agent preferentially segregates towards the air-coating surface of the PU primer formulation at higher curing temperature. However, given that the concentration of nitrogen for both PU primers is similar for PMTs above $232^{\circ} \mathrm{C}$, it would seem that any variations in surface elemental composition observed are minor for PU primer coatings cured at or above this temperature. A similar finding has been reported by Perruchot et al. for the effects of variation of the PMT employed during curing of a series of polyester coil coating formulations. [17] With knowledge of this result all primer coatings, regardless of formulation, used in the studies described here for the investigation of PU primer to PVdF topcoat intercoat adhesion, were cured at a PMT of $232^{\circ} \mathrm{C}$.

The five PU primer formulation variations described in Table 1 were applied to HDGS substrate, cured at $232^{\circ} \mathrm{C}$ and characterised by XPS. The surface elemental compositions (in atomic \%) for each of the PU primer formulations are presented in Table 4. An initial inspection of Table 4 reveals that the PU primers E1, E2, E3 and E4 possess similar concentrations of carbon at their surface. The E2 (crosslinker changed) and E4 (extra crosslinker and matting agent) PU primer surfaces exhibit 
similar concentrations of oxygen, while the oxygen concentration of the E3 (extra crosslinker) PU primer surface is somewhat lower and the oxygen concentration of the E1 PU primer surface somewhat higher than those observed for E2 and E4. However, the E1, E2, E3 and E4 oxygen concentration values fall within a narrow range. The similarity in the carbon and oxygen concentrations for E1, E2, E3 and E4 PU primer surfaces suggests the chemical compositions of these four surfaces are very similar.

The elemental composition of the E5 PU primer is markedly different to that of the other PU primers investigated, making it something of an anomaly. The E5 PU primer surface displays increased carbon and oxygen concentrations and a lower nitrogen concentration than the other PU primer coatings examined. Perhaps, most notably, no tin signal is observed at the E5 PU primer surface. Table 1 described the E5 PU primer formulation as containing an acrylic flow agent. It is a well reported phenomena that flow agents segregate to the surfaces of coating and paints [14-16] where they perform their principle function, namely, that of aiding flow by lowering the surface free energy of the pre-cured coating formulation. Often, flow agents are employed to perform additional functions such as leveling or anti-cratering, which improve the surface finish of a coating or paint. Perruchot et al., employing angle resolved XPS, have demonstrated that flow agents form a segregation layer at the surface of cured coatings, that are typically 0.5 to $2 \mathrm{~nm}$ thick [18] depending on the type and quantity of flow agent employed in the coating formulation. The increased carbon and oxygen concentrations and lower nitrogen concentration observed for the 
E5 PU primer surface in Table 4 are the result of the formation of an acrylic flow agent layer at the air-coating surface. By segregating to the surface and forming a layer, the flow agent strongly attenuates the nitrogen signal arising from the crosslinking resin. Furthermore, the signal arising from the presence of the organotin catalyst in the primer formulation is completely masked by the presence of the flow agent layer, thus leading to the lack of tin signal as observed in Table 4 for the E5 PU primer surface.

To investigate intercoat adhesion failure the PU primers, E1 to E5 (cured at $232^{\circ} \mathrm{C}$ ) were coated with a standard PVdF based topcoat formulation. The PVdF topcoat was cured at $249^{\circ} \mathrm{C}$ with an oven dwell time of 55 s directly onto the PU primer coating. To examine the surfaces resulting from adhesion failure between the two polymeric coatings, coated specimens were scored through to the substrate in a cross-hatch pattern with a scalpel. Where possible the PVdF topcoat was removed from the PU primer with tweezers. In some instances it was necessary to apply an adhesive tape peel to the scored cross-hatch so as to initiate delamination of the PVdF topcoat from the PU primer. Once delaminated, three surfaces were available for analysis: the PU primer adhesion failure surface, the PVdF topcoat adhesion failure surface and the PVdF topcoat air-coating surface. A schematic describing the delamination of the topcoat and the surfaces available for analysis is presented in Figure 2. The XPS elemental composition analysis results for the adhesion failure surfaces of the different PU primers, the corresponding PVdF topcoat adhesion failure surfaces and the PVdF air-coating surfaces are presented in Table 5. 
Comparison of the data from the primer sides of the failed interfaces in Table 5 with those of the pristine PU primers characterised in Table 4 indicates attenuation of the carbon, nitrogen and tin surface concentrations at the PU primer adhesion failure surfaces. An exception is the nitrogen content of the E5 PU primer adhesion failure surface, which exhibits an increased nitrogen concentration compared to that of the pristine surface in Table 4. It is also observed that the PU primer adhesion failure surfaces data in Table 5 exhibit a general increase in oxygen concentration when compared to the uncoated primers in Table 4. The most notable difference between the surface elemental compositions of the original primers in Table 4 and those of the PU primer adhesion failure surfaces in Table 5 is the appearance of fluorine on the PU primer side of the adhesion failure interface.

All fluorine data contained in Tables $4 \& 5$ is collated in Figure 3 to allow ready comparison of concentration of fluorine as a function of primer type and location within the coating system. All of the PU primer adhesion failure surfaces investigated exhibited a fluorine surface concentration in the range $2.5 \pm 0.4 \%$. Although the fluorine must originate from the disbonded PVdF topcoat, the exact nature of the fluorine has yet to be determined. The fluorine may exist as a monolayer covering the majority of the surface, as islands in regions of the PU primer where adhesion was particularly strong or may result from penetration of fluorine bearing components from the PVdF topcoat into the uppermost regions of the PU primer coating. 
XPS elemental composition data for the PVdF sides of the failed interfaces after delamination from each of the five PU primers investigated is also presented in Table 5. Figure 4 shows the XPS survey spectrum acquired from the PVdF topcoat side of the failed interface after delamination from the primer with an additional crosslinker (E3). XPS signals characteristic of C1s ( 285 eV), O1s ( $533 \mathrm{eV}), \mathrm{F} 1 \mathrm{~s}(\sim 686 \mathrm{eV})$ and F2s $(\sim 30 \mathrm{eV})$ are observed. Comparison of the elemental composition of this interface with that from the PVdF side of the failed interface from the primer with both extra crosslinker and matting agent (E4) highlights almost identical surfaces indicating that the mode of failure is the same and that the influence of the matting agent in the primer on adhesion comes from the increased surface roughness rather than any chemical interaction.

It is possible to get information on the mechanism of failure by comparing the spectra from the PVdF sides of the failed interfaces with those taken from the top PVdF surfaces provided it is assumed no segregation phenomena are taking place. The lack of nitrogen on the PVdF side of the interface and its reduced concentration on the primer side is in concert with reduced concentrations of $\mathrm{C}$ and $\mathrm{Sn}$, together with the presence of fluorine on that side $(\sim 2 \%)$ suggests that delamination occurs just into the PVdF side of the interface.

It was noted in the analysis of the XPS elemental composition data for the PU primer adhesion failure surfaces that the E5 PU primer (flow aid added) exhibited an 
increased surface nitrogen concentration $(\mathrm{N}=1.9 \%)$ upon removal of the PVdF topcoat, when compared to the pristine PU primer $(\mathrm{N}=0.8 \%)$. It is also observed in Table 5 that the PVdF side of the failed interface, complementary to the failed E5 PU primer interface exhibits an increased oxygen concentration and a decreased fluorine concentration when compared to the PVdF topcoats air-coating surface (see Table 5). These results suggest that as the PVdF topcoat is removed from the E5 PU primer some of the top surface of the primer (in effect the flow-agent layer described earlier) is stripped and transferred to the PVdF side of the interface. However the carbon and oxygen content is similar to that seen on the PVdF failure interfaces of E3 and E4 and may indicate that the flow aid is not there and that the weak boundary layer is not made up from this material. An alternative hypothesis is that the presence of the flow aid inhibits the inter-diffusion of the primer and topcoat components because its own dissolution process takes priority. Thus a substantial interphase region is prevented from forming during the brief cure schedule. The increased nitrogen concentration is due to a layer of the PU primer that is richer in nitrogen than the surface, being exposed by stripping off the flow agent layer when the PVdF topcoat is removed. The sub-surface nitrogen rich layer is due to the flow agent competing for the surface and inhibiting the segregation of the crosslinker to the surface, thus even when the flow agent is removed the nitrogen does not recover to the concentration seen in the other primers.

A further detailed look at the surface composition shows a reduced fluorine concentration at the PVdF top surface for E5 compared to the other 4 formulations. In 
addition the carbon content is considerably higher and the oxygen content significantly lower. These changes in $\mathrm{C}, \mathrm{O}$ and $\mathrm{F}$ concentration suggest that the flow agent in the primer has some effect on the topcoat. It supports the hypothesis that the flow agent dissolves in the liquid PVdF topcoat which contains solvents prior to baking and segregates to the air-coating interface in competition with the fluorine moieties that normally move to the same interface within the time scale of this baking schedule (10 s between application and oven, $45 \mathrm{~s}$ dwell time in the oven). The acrylic flow aid is more likely to segregate to the surface as the PVdF is present as discrete particles until they dissolve in the isophorone solvent at high temperature. However, the surface composition is significantly different to that of the primer with flow aid (E5) and so this requires further investigation with TOF-SIMS.

The XPS elemental composition data for the PVdF topcoat adhesion failure surfaces of the $\mathrm{E} 1(\mathrm{C}=62.2 \%, \mathrm{O}=15.2 \%, \mathrm{~F}=22.6 \%$, reference formulation $)$ and $\mathrm{E} 2(\mathrm{C}=$ $63.5 \%, \mathrm{O}=17.4 \%, \mathrm{~F}=19.1 \%$, new crosslinker) $\mathrm{PU}$ primer samples given in Table 5 are significantly different from those for the $\mathrm{E} 3(\mathrm{C}=58.9 \%, \mathrm{O}=12.0 \%, \mathrm{~F}=29.1 \%$, extra crosslinker) sample. For the E1 and E2 topcoat adhesion failure surfaces a considerable increase in carbon and oxygen concentrations is noted when compared to the PVdF topcoat air-coating surface (see Table 5). Additionally, a substantial decrease in the fluorine surface concentration is observed for the same failure interfaces, when compared to the PVdF topcoat air-coating surfaces (see Figure 3). These results clearly indicate transfer of non-nitrogen containing components from the PU primers E1 and E2 to the PVdF topcoats interfacial surface. However, a 
review of Table 1 indicates that the E1 and E2 primer formulations contain less of the nitrogen containing cross-linking agent used in the E3, E4 and E5 primer formulations. This suggests that the increased carbon and oxygen concentrations, observed at the PVdF adhesion failure surfaces for E1 and E2 PU primers, result from the transfer of non-crosslinked polyester resin material from the PU primer which arises from the reduced concentration of the crosslinking agent in these formulations. They again will be checked employing TOF-SIMS.

These findings clearly indicate that the degree of crosslinking achieved in the primer is extremely important in terms of the adhesive properties exhibited by the primers towards the PVdF topcoat. If the primer coating is under crosslinked, the rigidity and mechanical properties of the primer are compromised, and adhesion failure occurs on the PU primer side of the PU primer/PVdF topcoat interface due to the presence of a weak boundary layer consisting mainly of un-crosslinked polyester. If the primer is over crosslinked then inter-coating diffusion is reduced or eliminated and true interfacial failure will occur. These results are of particular importance to the coating formulator as they suggest that the balance of crosslinker resin concentration and cure conditions can have wide ranging effects on properties such as intercoat adhesion.

Consideration of the nitrogen concentration values presented in Table 4 for the pristine PU primer surfaces, percentage adhesion values from the cross hatch adhesive tape peel test given in Table 2 and the analysis of the PU primer and PVdF topcoat adhesion failure surfaces in Table 5 suggests a correlation between the 
concentration of nitrogen at the PU primer surface and the strength of adhesion exhibited by the PU primer towards the PVdF topcoat. The E3 and E4 PU primer coatings (both of which possess a nitrogen concentration $>3 \%$, see Table 4) display the greatest adhesion towards the PVdF topcoat $(75 \%$ and $90 \%$ adhesion respectively, see Table 2) of the PU primer formulations investigated. Conversely, the E5 PU primer coating, which possesses the lowest concentration of nitrogen at its surface $(<1 \%$ in Table 4$)$ exhibits the poorest adhesion $(0 \%$ adhesion, see Table 2$)$ of all the PU primer formulations examined. The E1 and E2 PU primer coatings exhibit a strength of adhesion somewhat intermediate between the E3/E4 primers and the E5 version $[\mathrm{E} 1=38 \% \& \mathrm{E} 2=39 \%$ adhesion (see Table 2$)]$. These results are associated with nitrogen concentrations $>2 \%$ but $<3 \%$ at the PU primer surface.

Analysis of Table 1 indicates that the E1 and E2 PU primer formulations contain reduced concentrations of the nitrogen containing crosslinking resin, and presumably this leads to the lower nitrogen concentrations observed for these primer surfaces detailed in Table 4. Comparison of those nitrogen concentrations obtained for the higher crosslinking resin concentration formulations (E3 and E4) with those measured for the lower crosslinking resin concentration formulations (E1 and E2) suggests the excess crosslinking resin employed may account for as much as a third of the nitrogen signal observed at the PU primer surface. These results suggest the availability of nitrogen containing species at the PU primer surface plays an important role in the adhesive interactions displayed by the PU primer towards the PVdF topcoat. High PU primer adhesion strengths are correlated with increased nitrogen concentration at the 
PU primer surface. Phenomena that lower the nitrogen concentration at the PU primer surface, such as the segregation of flow agent to the coating surface or the reduction of nitrogen containing additives/components such as the crosslinking agent, lessen the adhesive properties exhibited by the PU primer towards the PVdF topcoat.

\subsection{Conclusions.}

We have characterised, by XPS, five variations of a polyester/polyurethane based primer formulation. We have also characterised by XPS the PU primer and PVdF topcoat adhesion failure surfaces and PVdF topcoat air-coating surface in studies encompassing intercoat adhesion failure. An initial study in which a subset of the PU primer formulations were cured at different PMTs demonstrated that beyond $232^{\circ} \mathrm{C}$ changes in PU primer surface composition are negligible. XPS characterisation of the pristine PU primers cured at $232^{\circ} \mathrm{C}$ indicated that inclusion of the matting agent in the E4 PU primer formulation had no effect on the surface composition observed and that the flow agent additive segregated to the surface of the E5 PU primer.

Analysis of the PU primer and PVdF topcoat adhesion failure surfaces indicates stripping of the segregated flow agent layer from the E5 sample and its transfer to the PVdF topcoat coating-coating surface. For the E3 and E4 PU primers, the transfer of quantities of components from the PVdF topcoat onto the primer and the lack of nitrogen on the backside of the PVdF upon delamination indicate the locus of 
adhesion failure is located on the PVdF side of the PU primer/PVdF topcoat interface. For the E1 and E2 PU primers, transfer of material from the PU primer to the PVdF topcoat is observed. This is the result of insufficient crosslinking of the polyester component, due to the reduced concentration of the crosslinking resin in these PU primer formulations.

PU primer adhesive properties are correlated with nitrogen concentration at the PU primer surface. Increased PU primer adhesion towards the PVdF topcoat is associated with higher nitrogen concentrations $(>3 \%)$ at the PU primer surface. These results are of particular significance to the coating formulator as they demonstrate that the inclusion or exclusion of components and additives that modify surface composition and chemistry may have a significant impact upon the properties exhibited by the coating, such as adhesion in the studies described here.

The presence of the flow aid on the surface of the E5 primer inhibits diffusion processes that would normally form a strong interphase region thus resulting in very weak intercoat adhesion. 


\section{Acknowledgements.}

The authors acknowledge the financial support of the EPSRC (Grant no.

GR/N65745).

\section{References.}

[1] Bongiovanni R, Montefusco F, Priola A, Macchioni, Lazzeri S, Sozzi L, Ameduri B, Progress in Organic Coatings 45 (2002) 359

[2] Barbucci A, Delucchi M, Cerisola G, Progress in Organic Coatings 30 (1997) 293.

[3] Seneviratne AMG, Sergi G, Page CL, Construction and Building Materials 14 (2000) 55.

[4] Cech V, Prikryl R, Balkova R, Vanek J, Grycova A, J. Adhesion Sci. Technol. 17 (2003) 1299.

[5] Nguyen TP, Le Rendu P, de Vos SA, Synthetic Metals 138 (2003) 113.

[6] Pesetskii SS, Jurkowski B, Kuzavkov AI, International Journal of Adhesion and Adhesives 18 (1998) 351.

[7] Haverkamp RG, Siew DCW, Barton TF, Surface and Interface Analysis 33 (2002) 330 .

[8] Bierwagen GP, Tallman DE, Progress in Organic Coatings 41 (2001) 201.

[9] Haack LP, Straccia AM, Holubka JW, Bhurke A, Xie M, Drzal LT, Surface and Interface Analysis 29 (2000) 829.

[10] Brady RF, Progress in Organic Coatings 43 (2001) 188.

[11] Adkins JD, Mera AE, Roe-Short MA, Pawlikowski GT, Brady RF, Progress in Organic Coatings 29 (1996) 1.

[12] Tritschler B, Forest B, Rieu J, Tribology International 32 (1999) 587.

[13] Fahlman M, Salaneck WR, Surface Science 500 (2002) 904.

[14] Leadley SR, Watts JF, Blomfield CJ, Lowe C, Surf. Interface Anal. 26 (1998) 444. 
[15] Watts JF, Abel M-L, Perruchot C, Lowe C, Maxted JT, White RG, J. Electron Spectrosc. Relat. Phenom. 121 (2001) 233.

[16] Perruchot C, Abel M-L, Watts JF, Lowe C, Maxted JT, White RG, Surf. Interface Anal. 34 (2002) 570.

[17] Perruchot C, Watts JF, Lowe C, Beamson G, International Journal of Adhesion and Adhesives 23 (2003) 101.

[18] Perruchot C, Watts JF, Lowe C, White RG, Cumpson PJ, Surf. Interface Anal. 33 (2002) 869.

[19] Li H, Chiba T, Higashida N, Yang Y, Inoue T, Polymer 38 (1997) 3921.

[20] Lowe C, Devos L, Lavaste V, Simmons GC, Watts JF, Journal of Coatings Technology 72 (2000) 135.

[21] Lauke B, Schüller T, Schneider K, Composite Interfaces 10 (2003) 1.

[22] Horgnies M, Darque-Ceretti E, Combarieu R, Progress in Organic Coatings 47 (2003) 154.

[23] Cho DL, Shin KH, Lee W-J, Kim D-H, J. Adhesion Sci. Technol. 15 (2001) 653. 


\begin{tabular}{|c|l|}
\hline Primer & Formulation Variation \\
\hline E1 & Base Reference Formulation \\
\hline E2 & $\begin{array}{l}\text { Base Reference Formulation \& change of crosslinking } \\
\text { agent }\end{array}$ \\
\hline E3 & As E2 \& an additional crosslinking agent \\
\hline E4 & As E3 \& matting agent \\
\hline E5 & As E3 \& acrylic flow agent \\
\hline
\end{tabular}

TABLE 1. PU primer formulation variations. 


\begin{tabular}{|c|c|}
\hline Primer & $\begin{array}{c}\text { Adhesion } \\
\text { Level / \% }\end{array}$ \\
\hline E1 & 38 \\
\hline E2 & 39 \\
\hline E3 & 75 \\
\hline E4 & 90 \\
\hline E5 & 0 \\
\hline
\end{tabular}

TABLE 2. PU primer grade denoting ease of PVdF Topcoat delamination from the PU primer. 


\begin{tabular}{|l|c|c|c|c|}
\hline Sample and PMT & \multicolumn{4}{|c|}{ Surface Composition / atomic \% } \\
& $\% \mathrm{C}$ & $\% \mathrm{~N}$ & $\% \mathrm{O}$ & $\% \mathrm{Sn}$ \\
\hline $\mathrm{E} 3 \mathrm{PMT}=224^{\circ} \mathrm{C}$ & 76.8 & 2.8 & 19.9 & 0.5 \\
\hline $\mathrm{E} 3 \mathrm{PMT}=232^{\circ} \mathrm{C}$ & 76.7 & 3.4 & 19.5 & 0.3 \\
\hline $\mathrm{E} 3 \mathrm{PMT}=242^{\circ} \mathrm{C}$ & 76.6 & 3.3 & 19.8 & 0.3 \\
\hline E3 PMT $=249^{\circ} \mathrm{C}$ & 76.5 & 3.4 & 19.9 & 0.2 \\
\hline E4 PMT $=224^{\circ} \mathrm{C}$ & 76.8 & 2.8 & 20.0 & 0.4 \\
\hline E4 PMT $=232^{\circ} \mathrm{C}$ & 76.2 & 3.2 & 20.2 & 0.4 \\
\hline E4 PMT $=242^{\circ} \mathrm{C}$ & 76.4 & 3.3 & 20.0 & 0.3 \\
\hline E4 PMT $=249^{\circ} \mathrm{C}$ & 76.5 & 3.5 & 19.7 & 0.3 \\
\hline
\end{tabular}

TABLE 3. Elemental composition of the polyurethane primer surfaces for formulations E3 \& E4 (in at. \%) cured at four different peak metal temperatures. 


\begin{tabular}{|c|c|c|c|c|}
\hline \multirow{2}{*}{ Primer } & \multicolumn{5}{c|}{ Surface Composition / atomic \% } \\
\hline E1 & $\% \mathrm{C}$ & $\% \mathrm{~N}$ & $\% \mathrm{O}$ & $\% \mathrm{Sn}$ \\
\hline E2 & $\mathbf{7 6 . 5}$ & $\mathbf{2 . 3}$ & $\mathbf{2 0 . 8}$ & $\mathbf{0 . 4}$ \\
\hline E3 & $\mathbf{7 6 . 8}$ & $\mathbf{2 . 6}$ & $\mathbf{2 0 . 2}$ & $\mathbf{0 . 4}$ \\
\hline E4 & $\mathbf{7 6 . 7}$ & $\mathbf{3 . 4}$ & $\mathbf{1 9 . 5}$ & $\mathbf{0 . 3}$ \\
\hline E5 & $\mathbf{7 6 . 2}$ & $\mathbf{3 . 2}$ & $\mathbf{2 0 . 2}$ & $\mathbf{0 . 3}$ \\
\hline
\end{tabular}

TABLE 4. XPS elemental compositions (in atomic\%) of the PU primer surfaces. A peak metal temperature of $232^{\circ} \mathrm{C}$ was employed to cure all PU primer samples investigated. 


\begin{tabular}{|c|c|c|c|c|c|}
\hline \multirow[t]{2}{*}{ Sample and Analysis Surface } & \multicolumn{5}{|c|}{ Surface Composition / atomic \% } \\
\hline & $\% \mathrm{C}$ & $\% \mathrm{~N}$ & $\% \mathrm{O}$ & $\% \mathrm{Sn}$ & $\% \mathrm{~F}$ \\
\hline E1 PU Failure Surface & 73.0 & 2.3 & 22.5 & 0.13 & 2.2 \\
\hline E1 PVdF Failure Surface & 62.2 & - & 15.2 & - & 22.6 \\
\hline E1 PVdF Top Surface & $\mathbf{5 5 . 5}$ & - & 10.9 & - & 33.6 \\
\hline E2 PU Failure Surface & 72.6 & 2.1 & 22.5 & - & 2.8 \\
\hline E2 PVdF Failure Surface & 63.5 & - & $\mathbf{1 7 . 4}$ & - & 19.1 \\
\hline E2 PVdF Top Surface & 54.8 & - & 11.7 & - & 33.5 \\
\hline E3 PU Failure Surface & 73.8 & 2.6 & 20.7 & $\mathbf{0 . 0 8}$ & 2.8 \\
\hline E3 PVdF Failure Surface & 58.9 & - & 12.0 & - & 29.1 \\
\hline E3 PVdF Top Surface & 55.4 & - & 10.7 & - & 33.9 \\
\hline E4 PU Failure Surface & 73.1 & 2.7 & 21.9 & 0.11 & 2.2 \\
\hline E4 PVdF Failure Surface & 58.6 & - & 12.8 & - & 28.6 \\
\hline E4 PVdF Top Surface & 55.3 & - & 11.2 & - & 33.6 \\
\hline E5 PU Failure Surface & 73.2 & 1.9 & 22.2 & - & 2.1 \\
\hline E5 PVdF Failure Surface & 58.2 & - & 11.9 & - & 29.9 \\
\hline E5 PVdF Top Surface & 59.4 & - & 8.8 & - & 31.9 \\
\hline
\end{tabular}

Table 5. Surface Compositions (in atomic \%) of the PU primer adhesion failure surface, the PVdF topcoat adhesion failure interface and the PVdF Topcoat air/coating surface. 


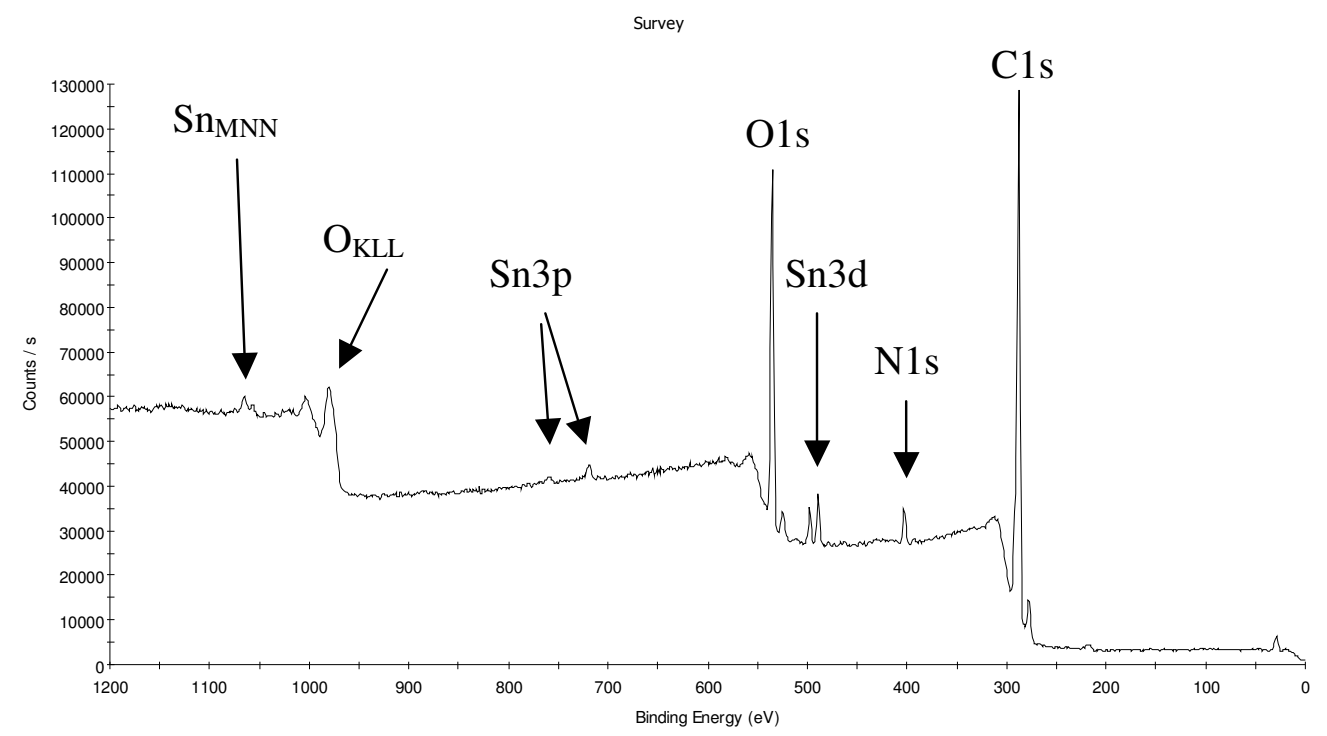

Figure 1. X-ray photoelectron spectroscopy survey spectra of the E3 PU primer coating formulation cured at a peak metal temperature of $224^{\circ} \mathrm{C}$. 


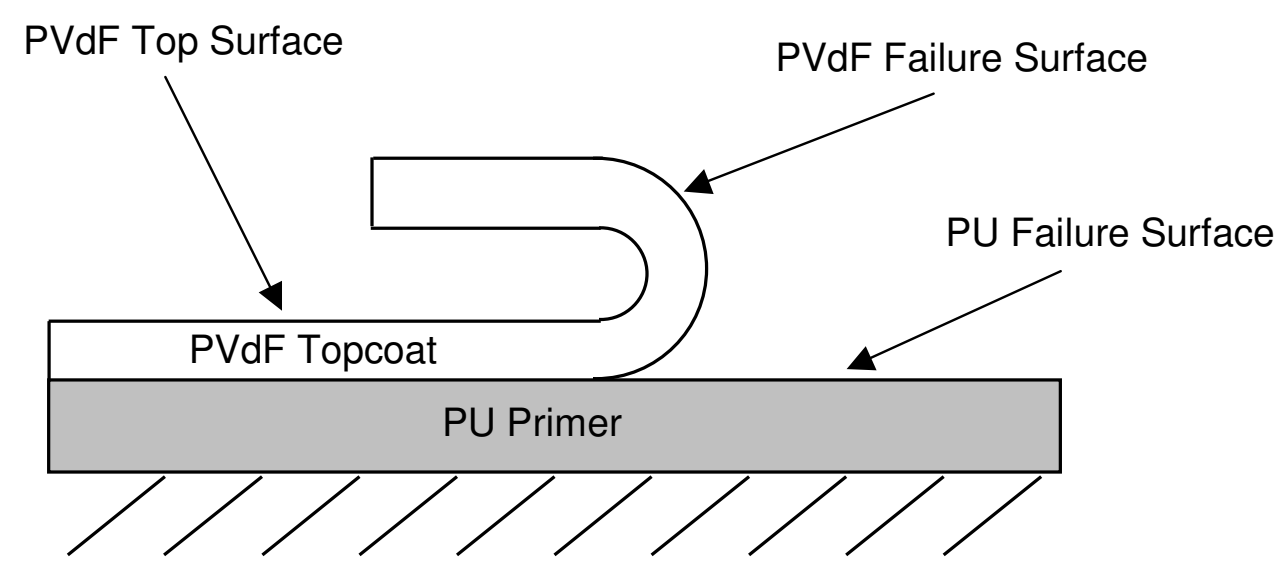

Figure 2. A schematic of the PU primer and PVdF topcoat intercoat adhesion failure surfaces and PVdF topcoat air-coating surface analysed. 


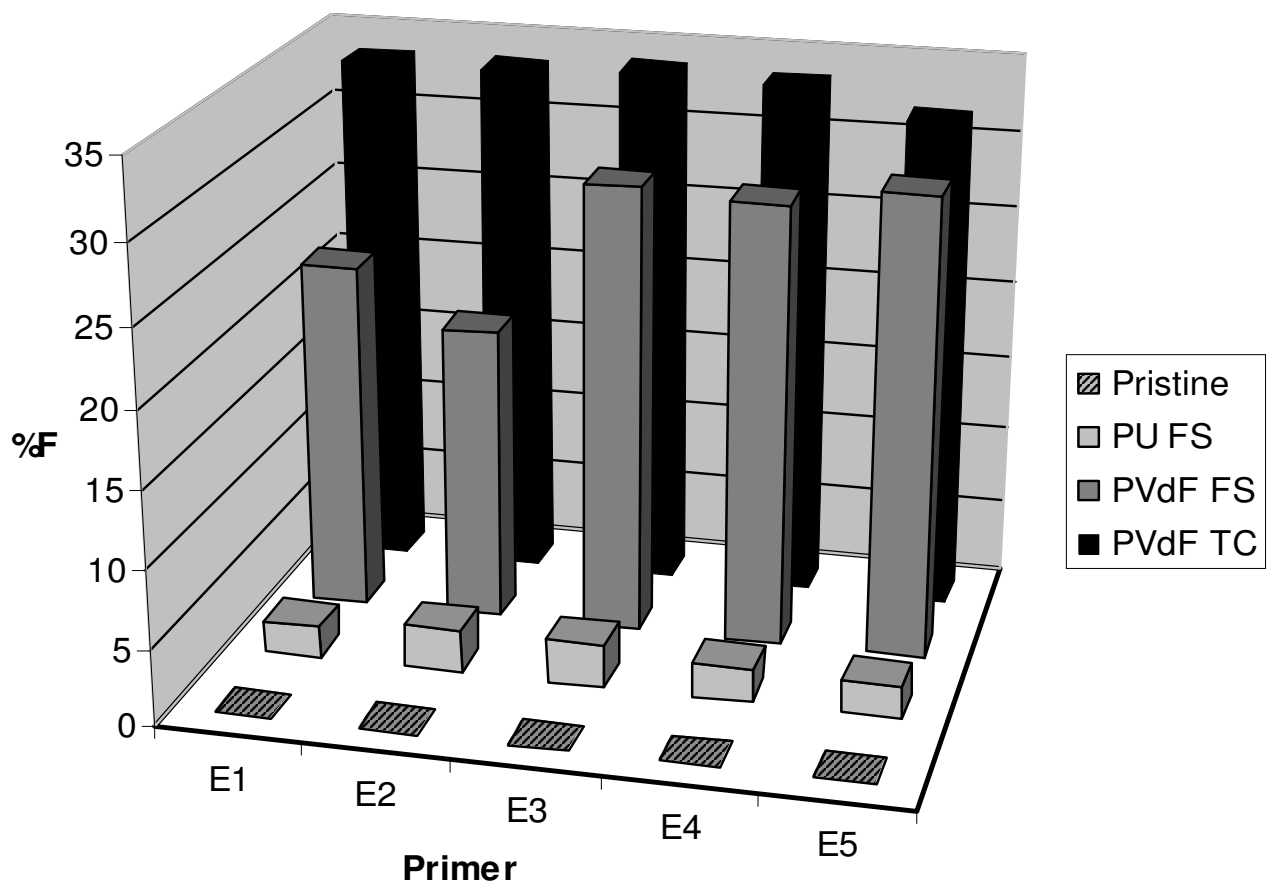

Figure 3. A 3-D histogram showing the fluorine concentrations of the pristine primer, the PU primer interfacial failure surface (PU FS), the PVdF topcoat interfacial failure surface (PVdF FS) and the PVdF topcoat air/coating surface (PVdF TC) for each of the PU primer formulations E1 to E5. 


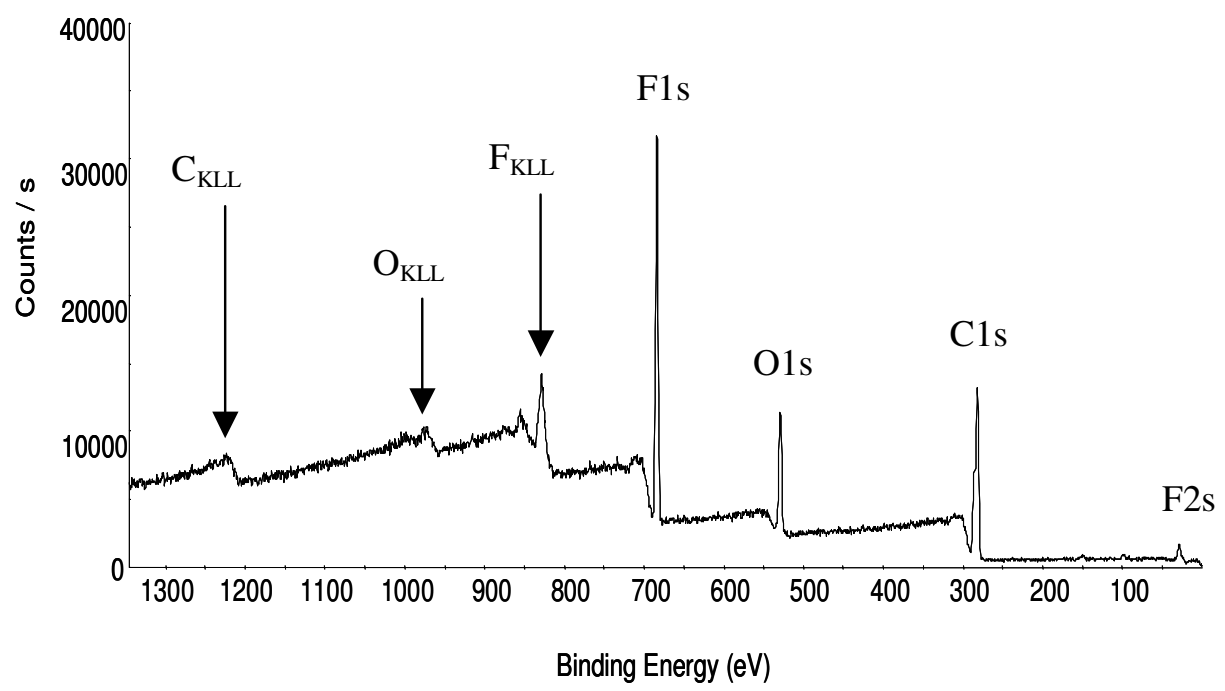

Figure 4. X-ray photoelectron spectroscopy survey spectra of the PVdF adhesion failure surface resulting from delamination of the PVdF topcoat from the PU primer formulation with an additional cross-linker (E3). 